\title{
FIRST COUNTABILITY \\ AND LOCAL SIMPLE CONNECTIVITY \\ OF ONE POINT UNIONS
}

\author{
KATSUYA EDA
}

(Communicated by Frederick R. Cohen)

\begin{abstract}
There exists a path-connected, simply connected, Tychonoff space $X$ with $x$ such that $X$ is locally simply connected at $x$, but $(X, x) \vee(X, x)$ is not simply connected.
\end{abstract}

H. B. Griffiths [1] proved,

Theorem 1 (H. B. Griffiths). Let $X$ be path-connected, locally simply connected at $x \in X$ and also first countable at $x$. Then, for an arbitrary path-connected space $Y$ with $y \in Y$, the fundamental group of the one point union $(X, x) \vee$ $(Y, y)$, i.e. $\pi((X, x) \vee(Y, y))$, is naturally isomorphic to the free product $\pi(X) * \pi(Y)$.

A question, which was commented in a footnote of [1, p. 176], is whether the first countability is essential in the statement. In this note we show that it is. Namely we prove,

Theorem 2. There exists a path-connected, Tychonoff space $X$ with $x \in X$ such that $X$ is locally simply connected at $X, \pi(X)$ is trivial but $\pi((X, x) \vee(X, x))$ is not trivial.

Since it seems that there is some confused understanding around this topic, e.g. [2, p. 146] and [3, p. 562], we explain an important point after some definitions. All spaces in this paper are Hausdorff. Undefined notions are standard [4]. We define $(X, X) \vee(Y, y)$ as the qoutient space of the topological sum of $X$ and $Y$ with the identification of $x$ and $y$ [2, p. 145] and call it a one-point union of $X$ and $Y$ with the common point $x=y$. A loop $f$ is a continuous map from a closed interval $[a, b]$ (where $a<b$ ) to a space such that $f(a)=f(b)$. For a subset $U$ of $X$, we say that a loop $f$ is in $U$ if the image of $f$ is included by $U$. Two loops $f$ and $g$ with the same domain $[a, b]$ are briefly said to be homotopic, if $f$ and $g$ are homotopic relative

Received by the editors May 16, 1989.

1980 Mathematics Subject Classification (1985 Revision). Primary 55Q20.

Key words and phrases. Fundamental group, one point union, locally simply connected, first countable. 
to $\{a, b\}$ [4]. If we do not make any specification, a loop is always a loop with its domain $[0,1]$. A space $X$ is locally simply connected at $x$, if for any neighborhood $U$ of $x$ there exists a neighborhood $V(\subset U)$ of $x$ such that any loop $f$ in $V$ is homotopic to a constant loop in $U$. A space $X$ is locally strongly contractible at $x$, if there exists a neighborhood $U$ of $x$ such that $i d_{U}$ and the constant map $x$ are homotopic relative to $\{x\}$. Let $Z$ be a one-point union of $X$ and $Y$ with the common point $x$. It is well-known and can be proved by a standard method that if $Z$ is locally simply connected at $x$ then $\pi(Z)$ is naturally isomorphic to $\pi(X) * \pi(Y)$. Suppose that $X$ and $Y$ are locally strongly contractible at $x$. Then, $Z$ is also locally strongly contractible at $x$ and hence $\pi(Z) \simeq \pi(X) * \pi(Y)$ naturally. The next proposition shows a role of first countability concerning homotopy and it also holds if $X$ is locally strongly contractible at $x$. We need a definition to state it. A loop $f$ in $(X, x) \vee(Y, y)$ with $f(0)=x$ is proper with respect to $X$, if $f$ satisfies the following: If $\left(a_{n}, b_{n}\right) \quad(n \in I)$ are pairwise disjoint open intervals such that $\bigcup_{n \in I}\left(a_{n}, b_{n}\right)=f^{-1}(X \backslash\{x\})$, then $I$ is finite.

Proposition 3 (essentially in [1, 2.1]). Let $X$ be locally simply connected at $x$ and first countable at $x$. Let $f$ be a loop in $(X, x) \vee(Y, y)$ with $f(0)=x$ such that $\bigcup_{n \in N}\left(a_{n}, b_{n}\right)=f^{-1}(X \backslash\{x\})$ and $\left(a_{m}, b_{m}\right) \cap\left(a_{n}, b_{n}\right)=\phi$ for $m \neq n$. Then, there exist $m \in N$ and a proper loop $f^{*}$ with respect to $X$ such that $f^{*}$ is homotopic to $f$ relative to $\left\{0,1, a_{n}, b_{n}: n \geq m\right\}$.

Since it is not so hard to prove this if we use the two given local properties and a similar proof can also be found in [3, Lemma 3.1], we omit the proof. Since Proposition 3 can be used effectively to prove Theorem 1, our strategy is to construct the desired space so that the conclusion of this proposition does not hold. Now we state the construction.

Let $X_{m}=\left\{(x, y):(x-1 / k)^{2}+y^{2}=1 / k^{2}, k \geq m\right\}$ for $m \in N$ and $Y_{m n}$ be spaces homeomorphic to

$$
Y_{m}=\left\{(x, y, z):(x-(1-z) / k)^{2}+y^{2}=(1-z)^{2} / k^{2}, k \geq m, 0 \leq z \leq 1\right\}
$$

for $m \in N$. Denote the homeomorphism from $Y_{m n}$ to $Y_{m}\left(\subset Y_{1}\right)$ by $i_{m n}$ and let $p_{m n}=i_{m n}^{-1}((0,0,1))$. Assume $Y_{m n} \cap Y_{m n^{\prime}}=X_{m}$ for each $m \in N$ and distinct $n, n^{\prime} \in N$, and $Y_{m n} \cap Y_{m^{\prime} n^{\prime}}=X_{m^{\prime \prime}}$ for distinct $m, m^{\prime} \in N$ and any $n, n^{\prime} \in N$, where $m^{\prime \prime}=\max \left\{m, m^{\prime}\right\}$. Let $X=\bigcup_{m, n \in N} Y_{m n}$. Next we define a topology on $X$. For each open subset $W$ of $X_{1}$ with $(0,0) \notin W$ and real $\epsilon>0$, let

$$
\begin{array}{r}
O_{W, \epsilon}=\left\{(x, y, z) \in Y_{1}: z<\epsilon \text { and }(x, y, z)\right. \text { is on the segment } \\
\text { between }(0,0,1) \text { and some point in } W\}
\end{array}
$$

and

$$
U_{W, \epsilon}=\bigcup_{m, n \in N} i_{m n}^{-1} O_{W, \epsilon}
$$


For each $m \in N$, open subset $W$ of $X_{1}$ with $X_{m} \subset W$, real $\epsilon>0$ and a $\operatorname{map} \varphi: N \rightarrow N$, let

$$
V_{W, \epsilon}^{\varphi, m}=\bigcup_{k \geq m \& n \geq \varphi(k)} Y_{k, n} \cup \bigcup_{k \geq m \& n<\varphi(k)} i_{k n}^{-1} O_{W, \epsilon} \cup \bigcup_{k<m \& n \in N} i_{k n}^{-1} O_{W, \epsilon} .
$$

The topology of $Y_{m n} \backslash X_{m} \quad(m, n \in N)$ is induced by $i_{m n}$. The open neighborhood bases of $(x, y) \in X_{1}$ with $(x, y) \neq(0,0)$ are $U_{W, \epsilon}$ and the open neighborhood bases of $(0,0)$ are $V_{W, \epsilon}^{\varphi, m}$. It is easy to check that the above defines a topology on $X$.

Lemma 4. Let $P=\left\{p_{m n}: m, n \in N\right\}$ and $x_{n} \in P$ for $n \in N$. If the sequence $x_{n}(n \in N)$ of members of $P$ converges, then there exists $p \in P$ such that $x_{n}=p$ for almost all $n \in N$.

Proof. Suppose not. By taking a subsequence, we may assume that $x_{k}(k \in N)$ are pairwise distinct. Let $x=\lim _{k \rightarrow \infty} x_{k}$. Clearly, $x$ must belong to $X_{1}$. If $x \neq(0,0)$, take an open $W \subset X_{1}$ so that $x \in W$ and $(0,0) \notin W$. Then, $U_{W, 1 / 2} \cap P=\phi$, which contradicts $\lim _{k \rightarrow \infty} x_{k}=x$. If $x=(0,0)$, we consider the two cases.

Case 1. There exists $m \in N$ such that $\left\{x_{k}: k \in N\right\} \subset\left\{p_{j n}: j<m, n \in N\right\}$. Take an arbitrary $\varphi: N \rightarrow N$. Then, $V_{X_{1}, 1 / 2}^{\varphi, m} \cap\left\{x_{k}: k \in N\right\}=\phi$, which is a contradiction.

Case 2. Otherwise. Then, there exists a subsequence $x_{i_{k}}(k \in N)$ such that $x_{i_{k}}=p_{m_{k} n_{k}}$ with $m_{k}<m_{k+1}$. Take $\varphi: N \rightarrow N$ so that $\varphi\left(m_{k}\right)>n_{k}$ for $k \in N$. Then, $V_{X_{1}, 1 / 2}^{\varphi} \cap\left\{x_{i_{k}}: k \in N\right\}=\phi$, which contradicts $\lim _{k \rightarrow \infty} x_{i_{k}}=(0,0)$.

Lemma 5. $X$ is a path-connected, Tychonoff space and locally simply connected at $x$.

Proof. Clearly, $X$ is path-connected. To see that $X$ is locally simply connected at $(0,0)$, take a neighborhood $V_{W, \epsilon}^{\varphi, m}$. We may assume that $\mathrm{W}$ is connected and $\left\{(x, y):(x-1 / i)^{2}+y^{2}=1 / i^{2}\right\} \not \subset W$ for $i<m$. Let $f$ be a loop in $V_{W, \epsilon}^{\varphi, m}$. Since $X$ is locally path connected, we may assume $f(0)=(0,0)$. By Lemma 4, there exist at most finitely many points in $P$ which belong to the image of $f$. Let $p_{k n} \in P \cap f([0,1])$ and $f^{-1}\left(Y_{k n} \backslash X_{k}\right)=\bigcup_{i \in I}\left(a_{i}, b_{i}\right)$, where $\left(a_{i}, b_{i}\right) \cap\left(a_{j}, b_{j}\right)=\phi$ for $i \neq j$ and $f\left(a_{i}\right), f\left(b_{i}\right) \in X_{k}$. Then, only finitely many open intervals $\left(a_{i}, b_{i}\right)$ intersect with $f^{-1}\left\{p_{k n}\right\}$. Therefore, combining finitely many homotopies in $Y_{k n}{ }^{\prime} s$ we conclude that $f$ is homotopic in $V_{W, \epsilon}^{\varphi, m}$ to a loop $f_{1}$ in $V_{W, \epsilon}^{\varphi, m}$ such that $f_{1}([0,1]) \cap P=\phi$. Define $r: X \backslash P \rightarrow X_{1}$ by $r(x)=x^{*} \in X_{1}$ where $x \in Y_{m n} \backslash\left\{p_{m n}\right\}$ and $x$ is on the segment between $p_{m n}$ and $x^{*}$. The continuity of $r$ at $x \neq(0,0)$ is clear. Since $V_{W, \epsilon}^{\varphi, m} \cap X_{1}=$ $W=r\left(V_{W, \epsilon}^{\varphi, m}\right), \quad r$ is also continuous at $(0,0)$. Hence, $r$ is a retraction. As shown in the above proof, $r \mid V_{W, \epsilon}^{\varphi, m}$ is also a retraction to $W$. Using this we get a loop $f_{2}$ in $W$ which is homotopic to $f_{1}$ in $V_{W, \epsilon}^{\varphi, m}$. Since $Y_{m \varphi(m)} \subset V_{W, \epsilon}^{\varphi, m}$ 
and $W$ is connected and $\left\{(x, y):(x-1 / i)^{2}+y^{2}=1 / i^{2}\right\} \not \subset W$ for any $i<m$, we can conclude that $f_{2}$ is homotopic to the constant loop in $V_{W, \epsilon}^{\varphi, m}$ and so is $f$. For $x \neq(0,0)$ and a neighborhood $U$ of $x$, we get a continuous map $g: X \rightarrow[0,1]$ so that $g(x)=0$ and $g(y)=1$ for $y \notin U$. To see this conclusion also holds for $(0,0)$ and $V_{W, \epsilon}^{\varphi, m}$, let $\rho$ be the Euclidean metric on the plane and define $h: X \rightarrow[0,1]$ by:

$$
h(x)=\left\{\begin{array}{cl}
w+\rho((u, v),(0,0)), & \text { if } k<m \text { or } n<\varphi(k) \\
w / k+\rho((u, v),(0,0)), & \text { otherwise; } \\
\text { where } i_{k n}(x)=(u, v, w) . &
\end{array}\right.
$$

Then, $h$ is continuous and the desired continuous map can be easily gotten using $h$. Therefore, $X$ is a Tychonoff space.

Proof of Theorem 2. Let $X$ be the space constructed already and $x=(0,0)$. It suffices to show that $\pi((X, x) \vee(X, x))$ is not trivial. Denote the first component of $(X, x) \vee(X, x)$ by $X^{A}$, the second one by $X^{B}$ and the common point by $x^{*}$. Every notion with superscript $\ldots{ }^{A}$ or $\cdots^{B}$ corresponds to a notion for $X$ in an obvious way. Let $f$ be a loop in $X^{A} \cup X^{B}$ such that $f(0)=$ $f(1)=x^{*}$ and $f \mid\left[1 / 2^{m}, 3 / 2^{m+1}\right]$ is a winding of $\left\{(x, y):(x-1 / m)^{2}+y^{2}=\right.$ $\left.1 / m^{2}\right\}^{A}$ and $f \mid\left[3 / 2^{m+1}, 1 / 2^{m-1}\right]$ is a winding of $\left\{(x, y):(x-1 / m)^{2}+y^{2}=\right.$ $\left.1 / m^{2}\right\}^{B}$ for $m \in N$. Suppose that $f$ is homotopic to the constant loop. Then, by definition there exists a continuous map $H:[0,1] \times[0,1] \rightarrow X^{A} \cup X^{B}$ such that $H(0, t)=H(1, t)=H(s, 0)=x^{*}$ and $H(s, 1)=f(s)$ for $s, t \in[0,1]$. Let $O_{i}(i \in I)$ be open connected components such that $H^{-1}\left(X^{A} \backslash\left\{x^{*}\right\}\right)=$ $\bigcup_{i \in I} O_{i}$ and $O_{i} \cap O_{j}=\phi$ for $i \neq j$. Likewise, let $H^{-1}\left(X^{B} \backslash\left\{x^{*}\right\}\right)=\bigcup_{j \in J} P_{j}$.

Case 1. $\left\{m:\left(1 / 2^{m}, 3 / 2^{m+1}\right) \times\{1\} \subset O_{i}\right\}$ are finite for all $i$. We get $i_{k} \in$ $I(k \in N)$ such that $i_{k} \neq i_{k^{\prime}}$ for $k \neq k^{\prime}$ and $\left\{m:\left(1 / 2^{m}, 3 / 2^{m+1}\right) \times\{1\} \subset\right.$ $\left.O_{i_{k}}\right\} \neq \phi$. For $k \in N$, let $H_{k}(\alpha)=H(\alpha)$ for $\alpha \in O_{i_{k}}$ and $H_{k}(\alpha)=x^{*}$, otherwise. Then, $H_{k}$ induces a homotopy from finite nontrivial windings to the constant loop. Therefore, there exist $\alpha_{k} \in O_{i_{k}}$ such that $H\left(\alpha_{k}\right)=p_{m_{k} n_{k}}^{A}$. Since $O_{i_{k}}(k \in N)$ is pairwise disjoint, there exists a condensation point $\alpha^{*}$ of $\left\{\alpha_{k}: k \in N\right\}$. Since we can get a subsequence of $\alpha_{k}(k \in N)$ which converges to $\alpha^{*}$, we assume $\lim _{k \rightarrow \infty} \alpha_{k}=\alpha^{*}$ for simplicity of notation. Then, $p_{m_{k} n_{k}}^{4}$ converges to $H\left(\alpha^{*}\right)$. Lemma 4 implies $H\left(\alpha^{*}\right) \in P$. On the other hand, for each $k \in N$ there exists a point $\beta_{k}$ on the segment from $\alpha_{k}$ to $\alpha^{*}$ such that $H\left(\beta_{k}\right)=x^{*}$. This implies $H\left(\alpha^{*}\right)=x^{*}$, which is a contradiction.

Case 2. $\left\{m:\left(1 / 2^{m}, 3 / 2^{m+1}\right) \times\{1\} \subset O_{i}\right\}$ is infinite for some $i$. Then, there exist $j_{k} \in J(k \in N)$ such that $j_{k} \neq j_{k^{\prime}}$ for $k \neq k^{\prime}$ and $\left\{m:\left(3 / 2^{m}, 1 / 2^{m-1}\right) \times\right.$ $\left.\{1\} \subset P_{j_{k}}\right\} \neq \phi$ by the connectedness of $O_{i}$. Now, we work in $X^{B}$ instead of $X^{A}$ and get a contradiction by the same argument as Case 1 . 
Remark. For spaces $X_{i}(i \in I)$ with $x_{i} \in X_{i}, \bigvee_{i \in I}\left(X_{i}, x_{i}\right)$ is the qoutient space of the topological sum of $X_{i}(i \in I)$ with the identification of all $x_{i} \quad(i \in I)$. We say that $\pi\left(\bigvee_{i \in I}\left(X_{i}, X_{i}\right)\right)$ is naturally isomorphic to $*_{i \in I} \pi\left(X_{i}\right)$, if the inclusion maps from $X_{i}$ to $\bigvee_{i \in I}\left(X_{i}, x_{i}\right)$ induce the isomorphism from $*_{i \in I} \pi\left(X_{i}\right)$ to $\pi\left(\bigvee_{i \in I}\left(X_{i}, x_{i}\right)\right)$. The crucial point is the surjectivity of the induced homomorphism. Remark that any loop $f$ in $\bigvee_{i \in I}\left(X_{i}, x_{i}\right)$ is in $\bigvee_{i \in F}\left(X_{i}, x_{i}\right)$ for some finite subset $F$ of $J$, since $X_{i}(i \in I)$ are Hausdorff. Therefore we get,

Proposition 6. Let $X_{i}(i \in I)$ be path-connected and $x_{i} \in X_{i}$. Suppose that $\pi\left(\bigvee_{i \in F}\left(X_{i}, x_{i}\right)\right)$ is naturally isomorphic to $*_{i \in F} \tau\left(X_{i}\right)$ for every finite subset $F$ of $I$. Then, $\pi\left(\bigvee_{i \in I}\left(X_{i}, x_{i}\right)\right)$ is naturally isomorphic to $*_{i \in I} \pi\left(X_{i}\right)$.

If $X_{i}$ is locally strongly contractible at $x_{i}$, or $X_{i}$ is locally simply connected at $x_{i}$ and also first countable at $x_{i}$, then the conclusion of Proposition 3 holds for $X_{i}$ and $x_{i}$ for each $i \in I$, as we mentioned before. Therefore, the assumption of Proposition 6 is satisfied in such a case.

\section{ACKNOWLEDGMENTS}

The author would like to say thanks to T. Kaneto and K. Sakai for stimulating conversations about this topic.

\section{REFERENCES}

1. H. B. Griffiths, The fundamental group of two spaces with a common point, Quart. J. Math. Oxford (2) 5 (1954), 175-190.

2. S. T. Hu, Homotopy theory, Academic Press, New York-London, 1959.

3. J. W. Morgan and I. A. Morris, A Van Kampen theorem for weak joins, Proc. London Math. Soc. (3) 53 (1986), 562-576.

4. E. H. Spanier, Algebraic topology, McGraw-Hill, New York and San Francisco, 1966.

Institute of Mathematics, University of Tsukuba, Tsukuba, Ibaraki 305, Japan 